\title{
Starkregen im urbanen Raum - Methoden und Modelle
}

\author{
Von Holger Hoppe und Daniela Falter
}

\section{Ausgangssituation und Veranlassung}

Nicht nur vor dem Hintergrund des zukünftig erwarteten Klimawandels und der prognostizierten Zunahme von Starkregenereignissen bedarf es kommunaler Anpassungsstrategien zur Vermeidung von Schäden durch Starkregenabflüsse in urbanen Gebieten. Starkregen führen schon heute zu erheblichen Sach- und Personenschäden. Auf Bundes-, Landes- und kommunaler Ebene wurden diese Herausforderungen inzwischen erkannt und entsprechende Strategien bzw. Förderprogramme entwickelt [1 bis 6$]$.

Die Art der zu entwickelnden Strategien hängt von der konkreten Aufgabenstellung ab und muss individuell abgestimmt werden. Im Fokus steht dabei häufig die Erstellung und Veröffentlichung von Starkregengefahrenkarten, wie sie z. B. die Städte Köln, Korschenbroich, Wuppertal und Oldenburg erarbeitet haben (s. Abschnitt 4).

Allen Aufgaben gemein ist jedoch das grundsätzliche Erfordernis umfassender (modellbasierter) Informationen zur Überflutungsgefährdung im urbanen Raum. Ohne entsprechende Informationen ist die Ermittlung wirksamer Anpassungsstrategien nicht möglich. Ansätze und Arbeitsschritte zur Ermittlung von Grundlagendaten zu Überflutungsgefahren sind im Merkblatt DWA M 119 „Risikomanagement in der kommunalen Überflutungsvorsorge für Entwässerungssysteme bei Starkregen“ [7] methodisch beschrieben. Zur Erstellung „urbaner Gefahrenkarten“ zum Thema Starkregen sind danach per Definition Wasserstände (und Fließgeschwindigkeiten) modelltechnisch zu ermitteln und darzustellen. Die Ermittlung entsprechender Daten gelingt ausschließlich unter Einsatz hydrodynamischer Modelle. Topografische Analysen allein reichen hier nicht aus.

Praxisbeispiele zeigen, dass inzwischen auch für größer Einzugsgebiete hydrodynamische Überflutungsbetrachtungen in hoher räumlicher Auflösung (1 m x $1 \mathrm{~m}$-Raster) erfolgreich durchgeführt werden können. Dabei kommen unterschiedliche Modellansätze zum Einsatz. Überflutungsgefahren können mittels hydrodynamischer Kanalnetzmodelle, Oberflächenabflussmodelle oder gekoppelter Kanalnetz- und Oberflächenabflussmodelle abgebildet werden.

Da die Ergebnisse der einzelnen Methoden strukturell sehr unterschiedlich sind und von verschiedensten Faktoren (zum Beispiel dem Ansatz zur Abflussbildung) beeinflusst werden, ist es wichtig, die Berechnungsmethode auf die jeweilige Aufgabenstellung abzustimmen. Weitergehende Erläuterungen zu diesem Themenfeld sind bisher im Regelwerk nicht beschrieben. Im Rahmen des von der Deutschen Bundesstiftung Umwelt geförderten Projekts KLASII „Entwicklung einer neuen Methodik zur vereinfachten, stadtgebietsweiten Überflutungsprüfung mit GIS-basierter Darstellung der Analyseergebnisse am Beispiel der Starkregenvorsorge und Klimaanpassung (KLAS) in Bremen“ [4] wurde daher eine Übersicht über relevante, aktuell in 
der Praxis zur Verfügung stehende Berechnungsmethoden erarbeitet und der Einfluss wichtiger Berechnungsfaktoren auf die Berechnungsergebnisse dargestellt.

Die Ergebnisse wurden in einem Praxisleitfaden veröffentlicht [8,9], der unter www.klasbremen.de zum Download bereit steht. Die folgenden Kapitel enthalten einen zusammenfassenden Auszug der Ergebnisse und zeigen weitere erfolgreiche Praxisbeispiele auf (Abschnitt 4).

\section{Grundlagen zu Kanalnetz- und Oberflächenabflussmodellen}

\subsection{Modellansätze}

Für die hydrodynamische Abbildung von Abflüssen im Kanalnetz und auf der Oberfläche werden heute sowohl Kanalnetzmodelle (1D), Oberflächenmodelle (2D) als auch gekoppelte Kanalnetz- und Oberflächenmodelle (1D/2D) eingesetzt.

\subsection{Grundlagendaten}

Die erforderlichen Grundlagendaten hängen dabei maßgeblich von der Berechnungsmethode und dem Modell ab. Der im Projekt KLAS erarbeitete Praxisleitfaden gibt einen Überblick über die jeweils erforderlichen Basisdaten und enthält konkrete Hinweise zum Aufbau von Kanalnetz- und Oberflächenmodellen im Kontext der Überflutungsmodellierung. Darüber hinaus werden Hinweise zur Auswahl einer für die jeweilige Aufgabenstellung und den jeweiligen Modellansatz geeigneten Niederschlagsbelastung gegeben. Thematisiert werden in diesem Zusammenhang Ziel- und Nachweisgrößen, die Art der Niederschlagsbelastung (Modellregen/Naturregen), der Beregnungsansatz (gleich- und ungleichmäßig) sowie die Dauer der Niederschlagsbelastung.

\subsection{Ansätze zur Abflussbildung}

Sowohl Kanalnetz- als auch Oberflächenmodelle beinhalten jeweils eigene Ansätze zur Abflussbildung. Werden beide Modell miteinander gekoppelt, muss vor der Berechnung entschieden werden, über welches Modell der effektive bzw. abflusswirksame Niederschlagsanteil berechnet werden soll. In Abbildung 1 sind Unterschiede zwischen den Ergebnissen der möglichen Modellkombinationen unter Berücksichtigung des Ansatzes zur Abflussbildung benannt. Zur eindeutigen Bezeichnung der Kombinationsmöglichkeiten wurde im Projekt KLAS eine Nomenklatur eingeführt, die sich aus den Elementen „Un“ für „ungekoppelt“, „Ge“ für „gekoppelt“, „K“ für „Kanalnetzmodell“ und „O“ für „Oberflächenmodell“ zusammensetzt (Bilder 1 und 2).

Je nach Aufgabenstellung sind auch kombinierte Modellansätze sinnvoll. Die im Forschungsprojekt KLAS entwickelte „kombinierte Methode“ als vereinfachter Ansatz zur stadtgebietsweiten Überflutungsprüfung ist $u$. a. auch bei der Erstellung der stadtgebietsweiten Starkregengefahrenkarten in Oldenburg und Wuppertal verwendet worden (Abschnitt 4). 
Dieser Ansatz ermöglicht die Berücksichtigung von Einflüssen aus dem Kanalnetz auch für sehr große Einzugsgebiete. Als Transport- und Speicherelement kann das Kanalnetz maßgeblich Einfluss auf Überflutungsprozesse haben und sollte daher bei der Ermittlung von Überflutungsgefahren in kanalisierten Einzugsgebieten berücksichtigt werden [10]. Liegen als Grundlage belastbarer Planungen keine aktuell fortgeschriebenen und kalibrierten hydrodynamischen Kanalnetzmodelle vor, sind kosteneffiziente Planungen nicht möglich.

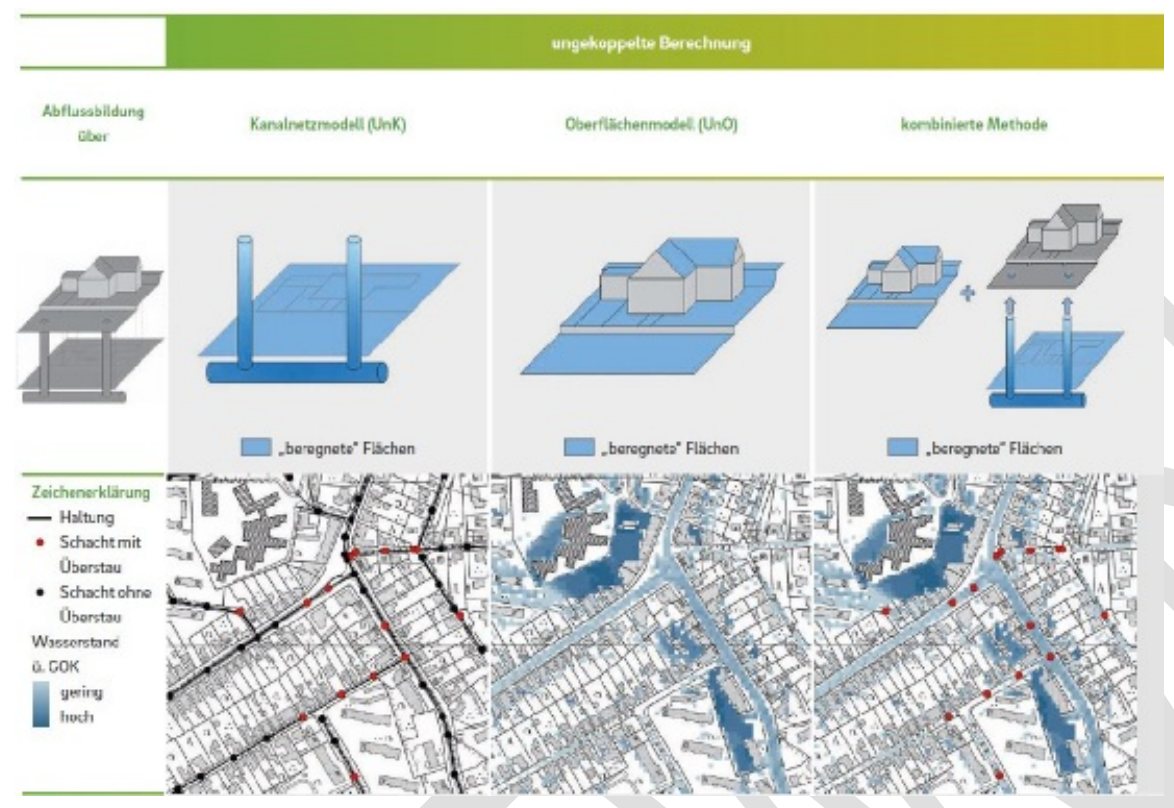

Bild 1: Übersicht über mögliche Berechnungsmethoden in Abhängigkeit der Abflussbildung - ungekoppelte Berechnungsansätze und kombinierte Methode zur Berücksichtigung des Überstaus [8]

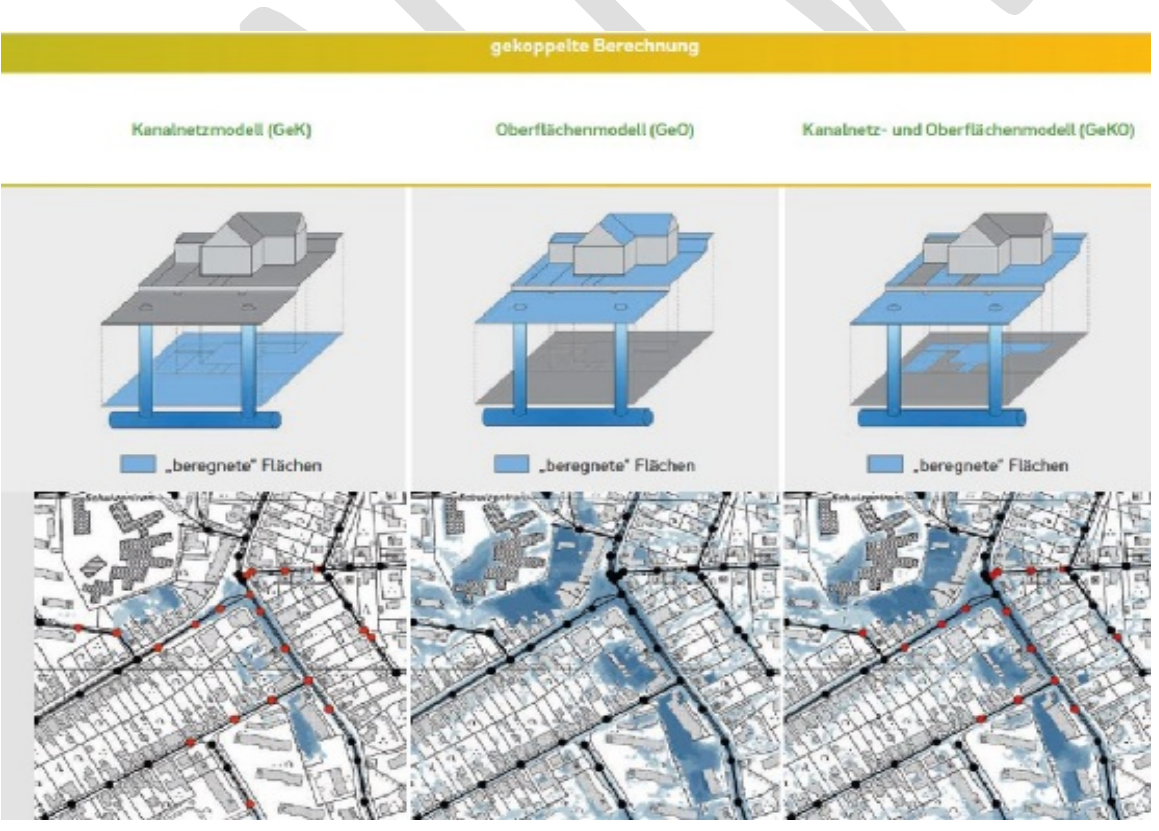

Bild 2: Übersicht über mögliche Berechnungsmethoden in Abhängigkeit der Abflussbildung - bidirektional gekoppelte Berechnungsansätze [8] 


\subsection{Ausgewählte Einflussfaktoren auf die Modellergebnisse}

Der Ansatz zur Abflussbildung ist jedoch nur ein Faktor von vielen, der Auswirkungen auf die Berechnungsergebnisse hat. Weitere wichtige Faktoren sind:

- die Struktur/räumliche Auflösung des Oberflächenmodells,

- die (exakte) Abbildung von Durchlässen und Verrohrungen,

- die Abbildung von Gebäude- und Mauerbruchkanten,

- die Berücksichtigung von Straßenabläufen sowie

- die Abbildung von Außengebieten,

- der Umgang mit unterschiedlichen Schachtdeckelniveaus,

- die Berücksichtigung von Interaktionen mit Gewässerabflüssen (Hochwasser).

\section{Vorgehensweise zur Auswahl einer Berechnungsmethode}

Akteure, die sich mit Fragen der Überflutungsvorsorge bzw. Klimafolgenanpassung beschäftigen, stehen bei der Entscheidung für eine konkrete Berechnungsmethode vor einer schwierigen Aufgabe. Daher wird ein schrittweises Vorgehen empfohlen, um ausgehend von der jeweiligen Fragestellung eine geeignete Berechnungsmethode auszuwählen [8].

\section{Schritt 1: Zieldefinition}

Der erste Schritt zur Auswahl der Berechnungsmethode besteht in der Festlegung der Ziele der angestrebten Überflutungsbetrachtung. Mit der Beantwortung der folgenden Fragen lässt sich ein erstes Anforderungsprofil für die Berechnung erstellen.

1. Was ist die Aufgabenstellung?

2. Welche Berechnungsergebnisse werden benötigt?

3. Welche Grundlagendaten und Modelle liegen mit welcher Qualität vor?

4. Welche Abflussprozesse sollen abgebildet werden?

5. Wie groß ist das zu betrachtende Gebiet?

6. Welche räumliche Auflösung der Ergebnisse ist aktuell und zukünftig erforderlich?

\section{Schritt 2: Auswahl eines/einer Modell/-kombination}

Mithilfe der Erkenntnisse aus Schritt 1 kann im zweiten Schritt die Auswahl einer Modellkombination und die eines Ansatzes zur Abflussbildung vorgenommen werden. Als Hilfsmittel steht hierbei die in Bild 1 und 2 dargestellte Übersicht über mögliche Berechnungsmethoden in Abhängigkeit der Abflussbildung zur Verfügung.

Schritt 3: Auswahl der Randbedingungen bzw. Einflussfaktoren Ist eine Berechnungsmethode ausgewählt, sollte über Art und Umfang der modelltechnischen Abbildung weiterer Einflussfaktoren (Bruchkanten, Mauern, Straßenabläufe, Außengebiete, Modellparameter, Berechnungsvarianten etc.) entschieden werden. 


\section{Praxisbeispiele zur stadtgebietsweiten Modellierung von Starkregen}

\subsection{Starkregengefahrenkarte Köln}

Einzugsgebiet: $\mathrm{rd} .400 \mathrm{~km}^{2}$

Modellansatz: Oberflächenabflussberechnung (UnO)

Auflösung: $1 \times 1 \mathrm{~m}$

Homepage: http://www.hw-karten.de/index.html?Module=Starkregen

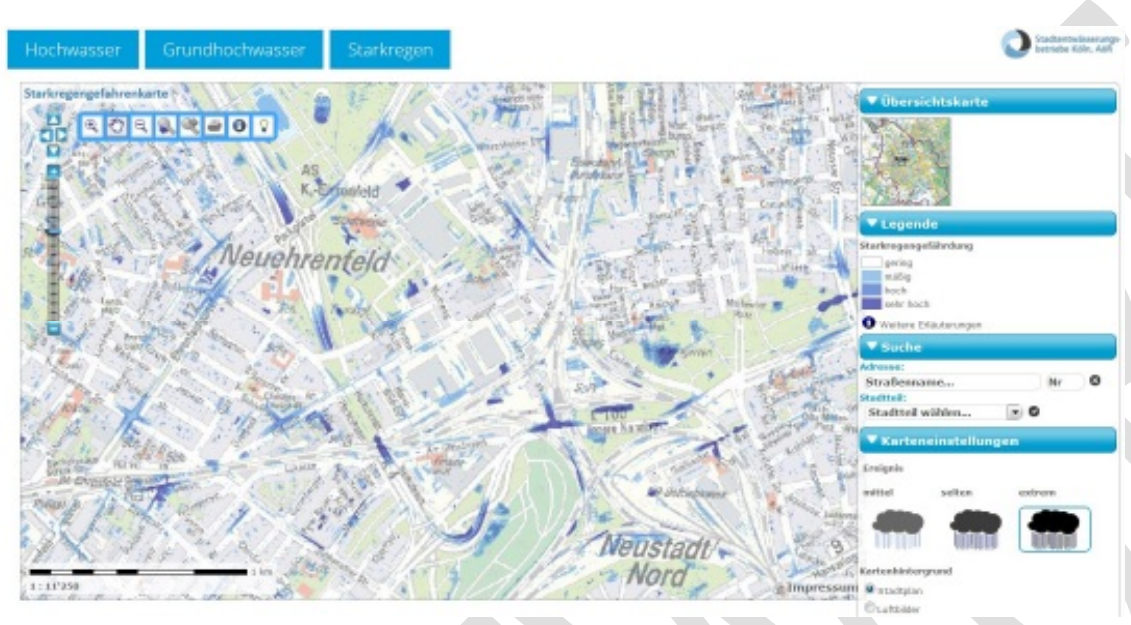

Bild 3: Auszug der Starkregengefahrenkarte Köln (Quelle: STEB Köln)

\subsection{Starkregengefahrenkarte Oldenburg}

Einzugsgebiet: $\mathrm{rd} .105 \mathrm{~km}^{2}$

Modellansatz: Kombinierte Methode (Berücksichtigung von Überstau)

Auflösung: $\quad 2 \times 2 \mathrm{~m}$

Homepage: https://gis4ol.oldenburg.de/Starkregengefahrenkarte

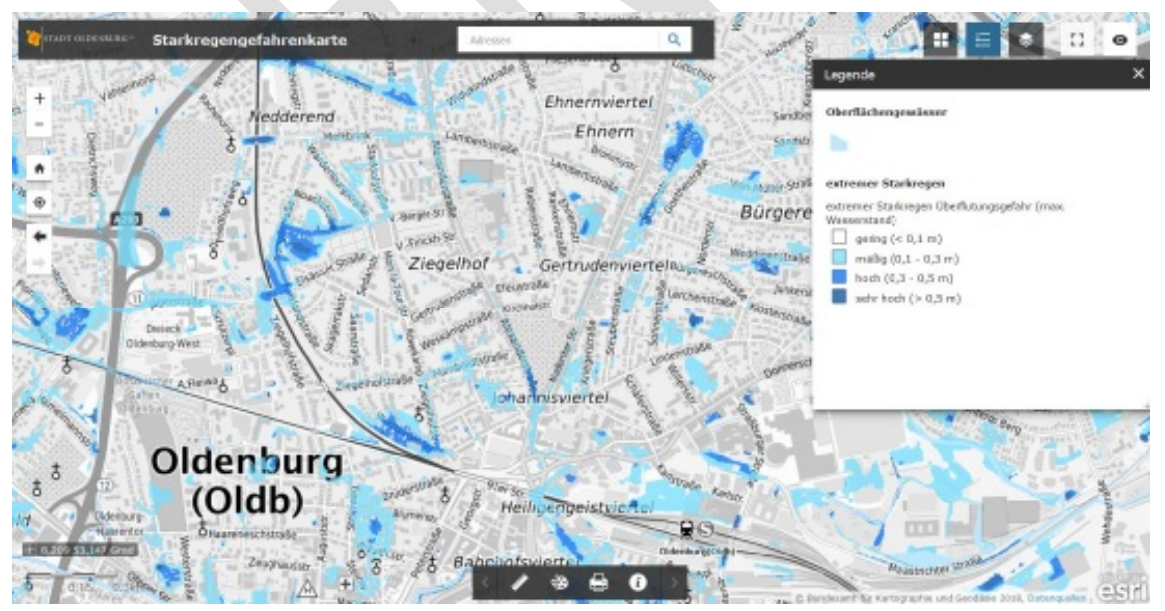

Bild 4: Auszug der Starkregengefahrenkarte Oldenburg (Quelle: Stadt Oldenburg) 


\subsection{Starkregengefahrenkarte Korschenbroich}

Einzugsgebiet: $r d .55 \mathrm{~km}^{2}$

Modellansatz: Kombinierte Methode (Berücksichtigung von Überstau)

Auflösung: $2 \times 2 \mathrm{~m}$

Homepage: www.korschenbroich.de/buergerservice/HochwasserundUeberflutungsschutz.php

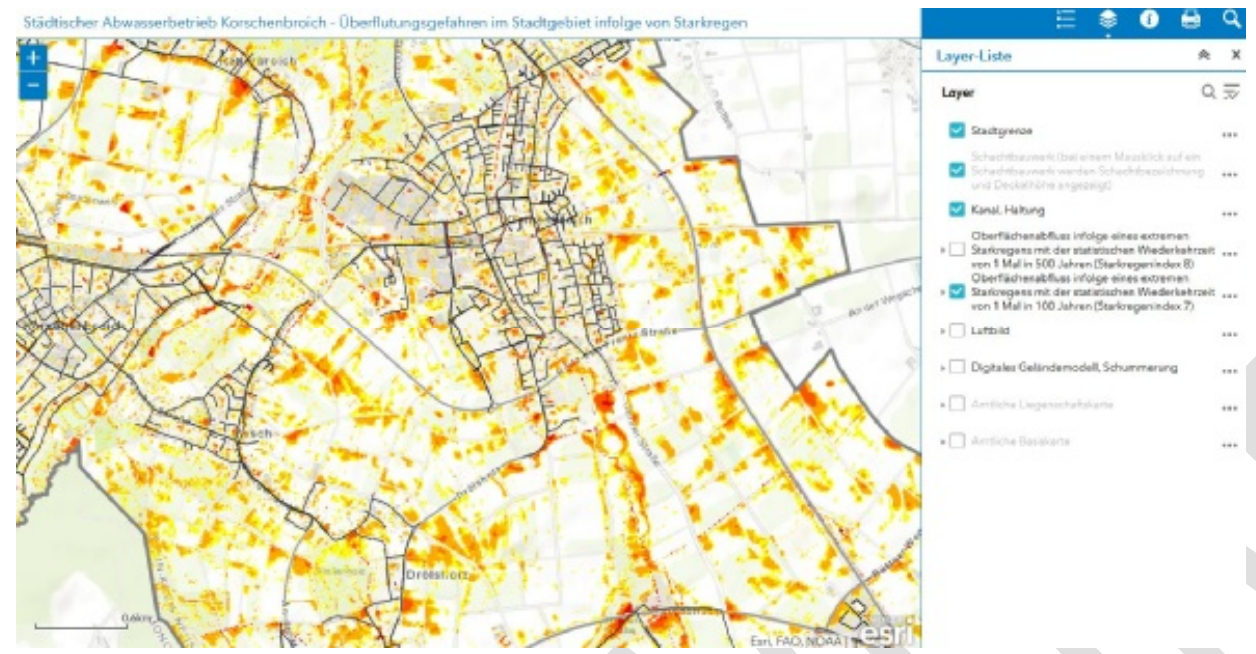

Bild 5: Auszug der Starkregengefahrenkarte Korschenbroich (Stadt Korschenbroich, Städtischer Abwasserbetrieb)

\subsection{Starkregengefahrenkarte Wuppertal}

Einzugsgebiet: rd. $235 \mathrm{~km}^{2}$

Modellansatz: Kombinierte Methode (Berücksichtigung von Überstau)

Auflösung: $1 \times 1 \mathrm{~m}$

Homepage: Veröffentlichung seit 12.2018 (Homepage Stadt Wuppertal und WSW AG)

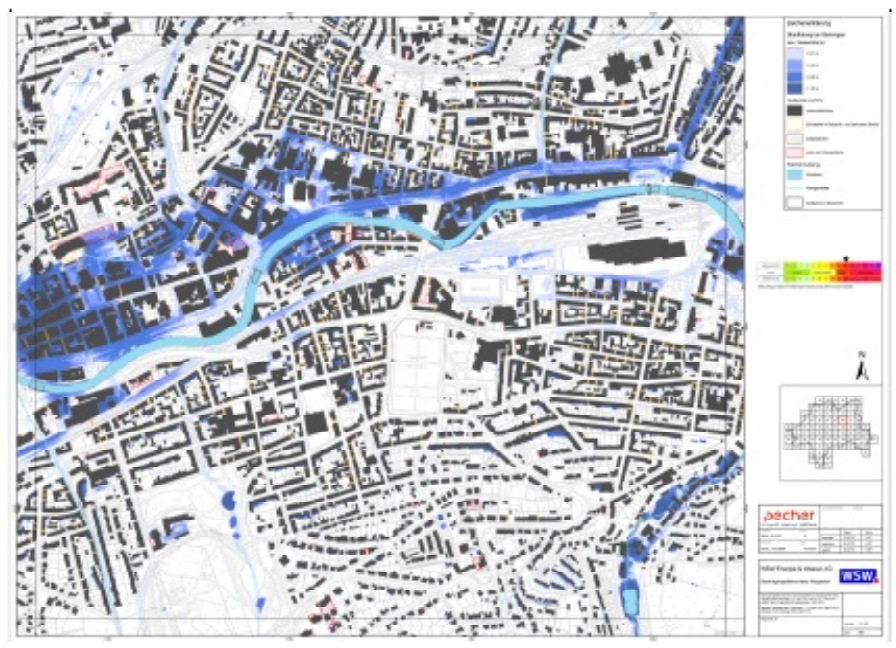

Bild 6: Grundlagen zur Starkregengefahrenkarte Wuppertal (Dr. Pecher AG/WSW/Stadt Wuppertal) 


\subsection{Belastungskarte Starkregen Landeshauptstadt Düsseldorf}

Einzugsgebiet: $\mathrm{rd} .220 \mathrm{~km}^{2}$

Modellansatz: Oberflächenabflussberechnung (UnO)

Auflösung: $\quad 5 \times 5 \mathrm{~m}$

Homepage: https://www.duesseldorf.de/kanal/kunden/starkregen-ueberflutungsschutz.html

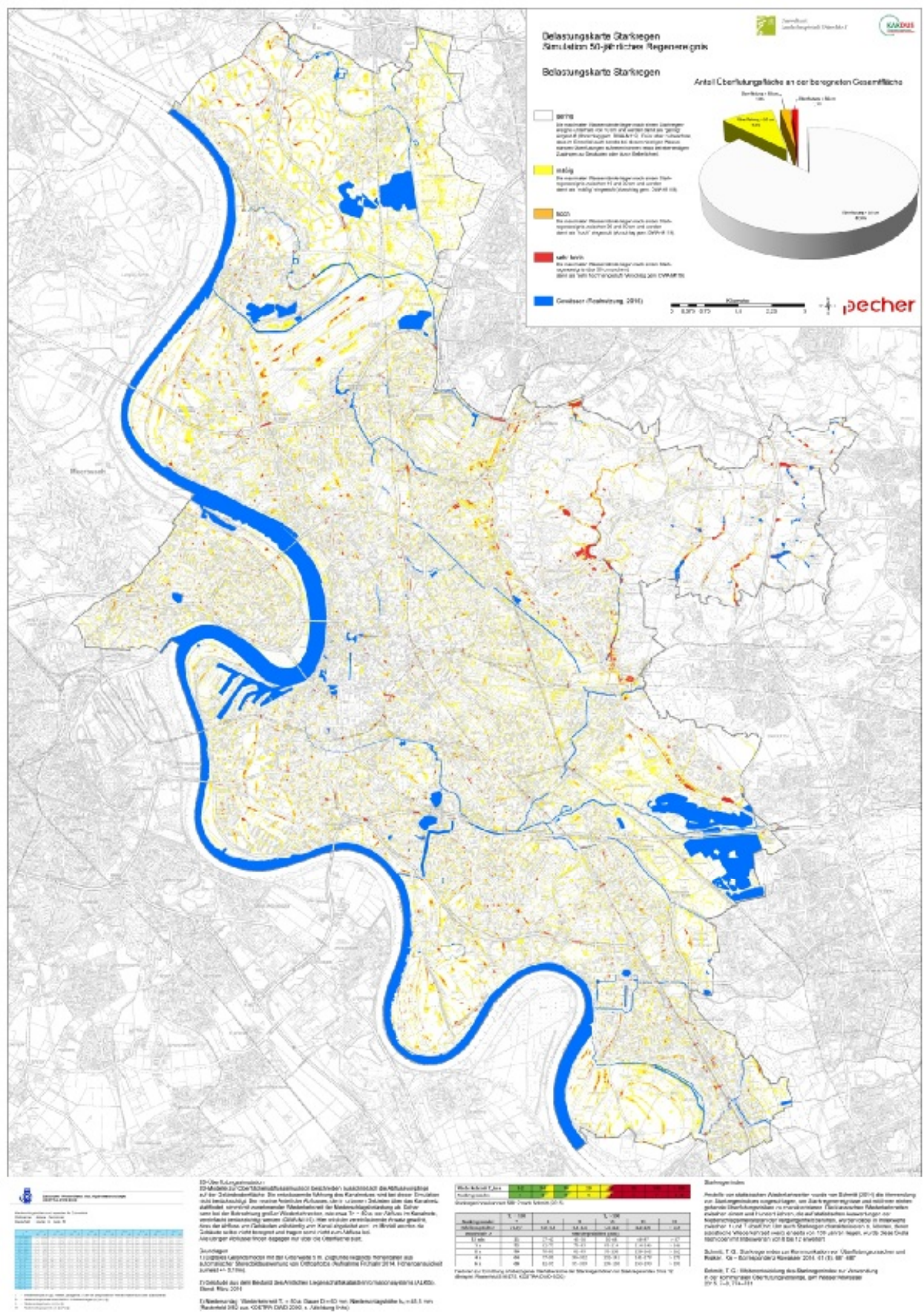

Bild 7: Belastungskarte Starkregen (Quelle: Landeshauptstadt Düsseldorf, Projekt KAKDUS) 


\subsection{Auskunfts- und Informationssystem (AIS) Starkregen Bremen}

Einzugsgebiet: rd. $330 \mathrm{~km}^{2}$

Modellansatz: Kombinierte Methode (Berücksichtigung von Überstau)

Auflösung: $\quad 1 \times 1 \mathrm{~m}$

Homepage: Veröffentlichung in 2019 (Homepage SUBV Bremen/hanseWasser Bremen)

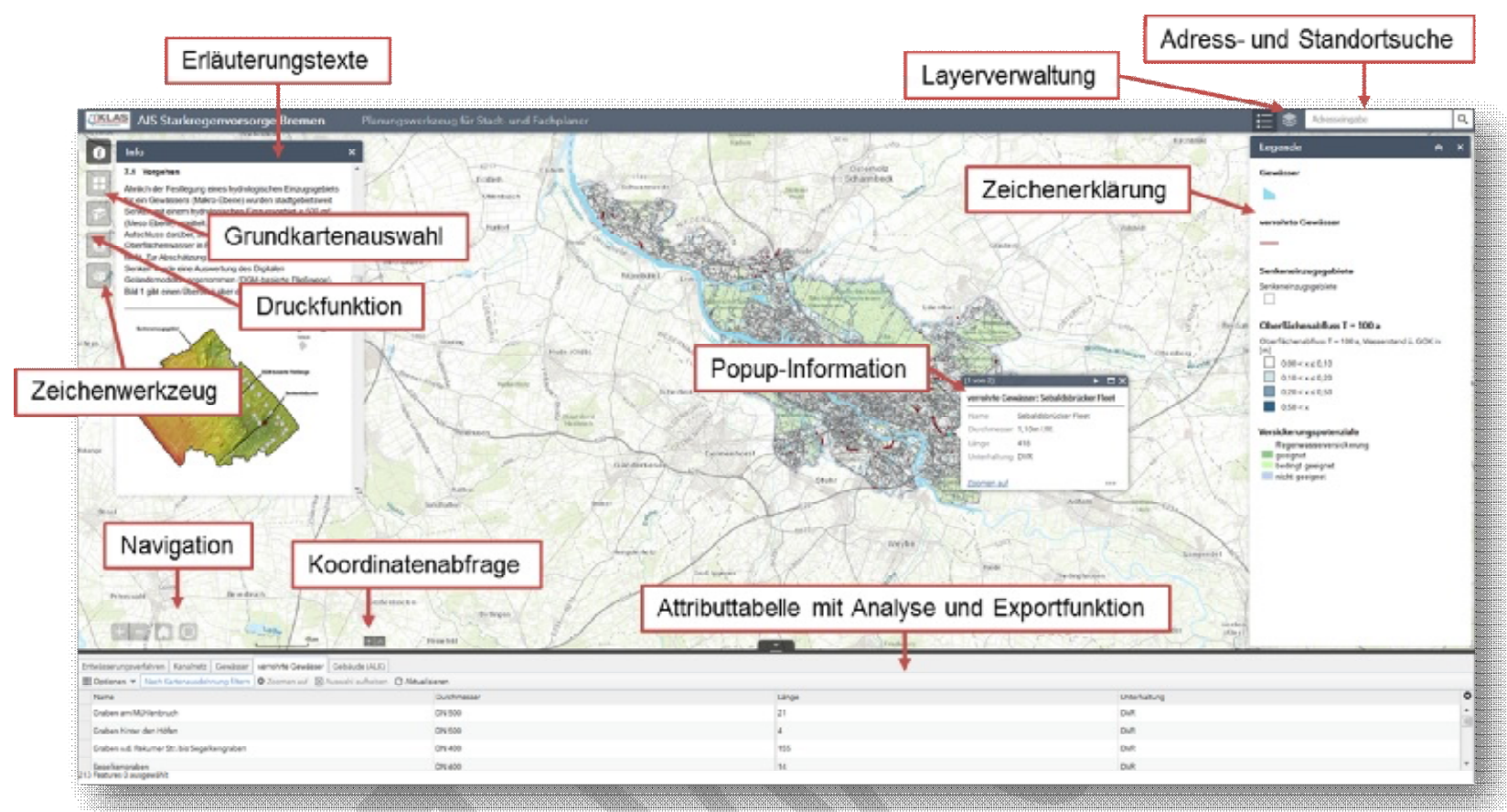

Bild 8: Entwurf Auskunfts- und Informationssystem Starkregen (AIS) Bremen (im Bild Ansichtsversion für die Stadtverwaltung in Bremen; Projekt KLAS Bremen)

\section{Zusammenfassung und Ausblick}

Starkregengefahrenanalysen in Form von stadtgebietsweiten Überflutungsmodellierungen stellen eine belastbare Grundlage für die weiteren Schritte des Starkregenrisikomanagements dar. Die Ausführungen des DWA-M 119 zum Risikomanagement in der kommunalen Überflutungsvorsorge für Entwässerungssysteme bei Starkregen ist methodisch wenig konkret. Der im Projekt KLASII entwickelte Praxisleitfaden knüpft hier an und bietet eine Übersicht über die aktuell in der Praxis gebräuchlichen Methoden zur hydrodynamischen Überflutungsberechnung.

Neben der ausführlichen Erklärung der Methoden und Ansätze von gekoppelten und ungekoppelten Kanalnetz- und Oberflächenmodellen werden auch Einflussfaktoren auf die Berechnungsergebnisse und benötigten Grundlagendaten angegeben. Das Ziel ist es, die entsprechenden Akteure der Überflutungsvorsorge bei der Auswahl geeigneter Methoden zu unterstützen. Dabei wird die Auswahl der Methoden in Relation zur jeweiligen Fragestellung gesetzt. 
Praxisbeispiele für stadtgebietsweite Überflutungsbetrachtungen, wie in Köln, Wuppertal oder Oldenburg, zeigen den aktuellen Umsetzungsstand in deutschen Kommunen zur Starkregengefahrenanalyse. Bremen, Düsseldorf und Bielefeld haben das Thema im Rahmen umfassender Klimaanpassungskonzepte mit betrachtet. Diese Analysen und Konzepte sind Grundlage für die weiteren Schritte des Starkregenrisikomanagements.

Auch auf Bundes- und Landesebene wurde der Bedarf an umfassenden Risikomanagementstrategien erkannt. Bestehende oder geplante Förderprogramme in Baden-Württemberg, Bayern oder NRW gehen über eine reine Gefahrenanalyse hinaus und fordern Strategien zum kommunalen Starkregenrisikomanagement. Hierzu zählen neben der Starkregengefahrenanalyse unter anderem auch Risikobetrachtungen, Maßnahmenplanungen und -priorisierungen sowie Beteiligungs- und Kommunikationskonzepte.

\section{Danksagung}

Der Aufsatz fasst wesentliche Ergebnisse des Forschungsprojekts KLASII „Entwicklung einer neuen Methodik zur vereinfachten, stadtgebietsweiten Überflutungsprüfung mit GIS-basierter Darstellung der Analyseergebnisse am Beispiel der Starkregenvorsorge und Klimaanpassung (KLAS) in Bremen“ (www.klas-bremen.de) zusammen. Ein besonderer Dank gilt der Deutschen Bundesstiftung Umwelt für die finanzielle Förderung und Begleitung des Vorhabens unter dem Aktenzeichen AZ32372/01 sowie allen Projektbeteiligten.

\section{Literatur}

[1] Bundesregierung. Fortschrittsbericht zur Deutschen Anpassungsstrategie an den Klimawandel. Stand: 16.11.2015 https://www.bmu.de/fileadmin/ Daten_BMU/ Download_PDF/Klimaschutz/klimawandel_das_fortschrittsbericht_bf.pdf (31. Oktober 2018)

[2] Ministerium für Umwelt, Landwirtschaft, Natur- und Verbraucherschutz des Landes Nordrhein-Westfalen. Hochwasserrisikomanagementplanung in NRW: Arbeitshilfe kommunales Starkregenrisikomanagement - Entwurf. Stand: Januar 2018. Unveröffentlicht

[3] Bayerisches Staatsministerium für Umwelt und Verbraucherschutz. Infoblatt zum Sonderprogramm: Integrale Konzepte zum kommunalen Sturzflut-Risikomanagement, 2017, https://www.stmuv.bayern.de/themen/wasserwirtschaft/ foerderung/doc/ infoblatt_sonderprogramm_sturzfluten.pdf (30. Oktober 2018)

[4] KlimaAnpassungsStrategie Extreme Regenereignisse (KLAS) - Umgang mit Starkregenereignissen in der Stadtgemeinde Bremen. Projektabschlussbericht, Der Senator für Umwelt, Bau und Verkehr, Freie Hansestadt Bremen, 2015, www.klasbremen.de (30. Oktober 2018)

[5] Landeshauptstadt Düsseldorf (Hrsg.) KAKDUS - Klimaanpassungskonzept für die Landeshauptstadt Düsseldorf - Kurzfassung. https://www.duesseldorf.de/ fileadmin/ Amt19/ umweltamt/ klimaschutz/ pdf/klimaschutz/ kurzfassung_klimaanpassungs konzept.pdf (30. Oktober 2018) 
[6] Umweltamt Bielefeld - Bielefeld fit für den Klimawandel. https://www.bielefeld.de /de/un/stkl/ (31. Oktober 2018)

[7] Merkblatt DWA-M 119: Risikomanagement in der kommunalen Überflutungsvorsorge für Entwässerungssysteme bei Starkregen, Hennef, 2016

[8] Ermittlung von Überflutungsgefahren mit vereinfachten und hydrodynamischen Modellen. Praxisleitfaden, erstellt im Rahmen des DBU-Forschungsprojekts „KLASII“, Lehrgebiet Siedlungswasserwirtschaft, Hochschule Bremen, 2017. www.klasbremen.de (30. Oktober 2018)

[9] von Horn J., Bonnet Ch., Schäfer K., Koch M., Thielking K., Gatke D., Jeskulke M. und Hoppe H. (2018). Ermittlung von Überflutungsgefahren mit vereinfachten und detaillierten hydrodynamischen Modellen - Ein Praxisleitfaden zu Begriffen, Berechnungsmethoden, Grundlagen und Einflussfaktoren. KA Korrespondenz Abwasser, Abfall 2018 (65), Nr. 2, S. 130ff. ISSN 1866-0029

[10] DWA: Stellungnahme zum Entwurf einer LAWA-Strategie für ein effektives Starkregenrisikomanagement (Stand: 4. September 2017), http://de.dwa.de/tl_files/_media/ content/ PDFs/Abteilung_WAW/ Hoe/Stellungnahmen/ DWA_Stellungn_Strategie\% 20Starkregen\% 20270917\%20fin.pdf (13. November 2017)

\section{Autoren:}

Dr. Holger Hoppe

Dr. Pecher AG

Klinkerweg 5

40699 Erkrath

Tel.: 0210493960

E-Mail:

holger.hoppe@pecher.de

Internet:

www.pecher.de

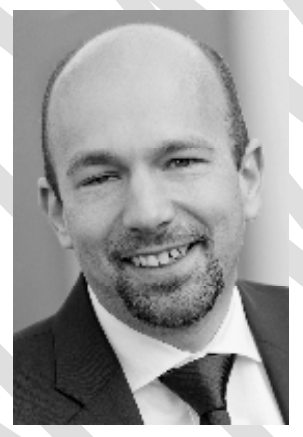

Dr. Daniela Falter

Dr. Pecher AG

Klinkerweg 5

40699 Erkrath

Tel.: 0210493960

E-Mail:

daniela.falter@pecher.de

Internet:

www.pecher.de

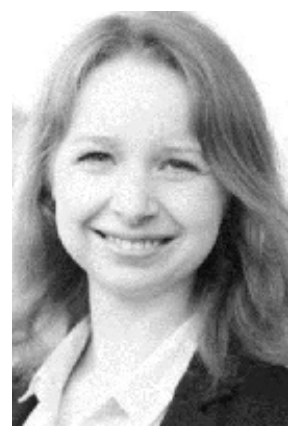

